\title{
La didáctica de la lengua en entornos virtuales de aprendizaje: el caso concreto de la enseñanza-aprendizaje del español como lengua extranjera y la plataforma Eleclips
}

\section{The didactics of language in virtual learning environments: the specific case of teaching-learning Spanish as a foreign language and the Eleclips platform}

\author{
Eva Álvarez Ramos \\ Universidad de Valladolid, Valladolid (España) \\ evamaria.alvarez.ramos@uva.es
}

\begin{abstract}
Resumen
La enseñanza de lenguas extranjeras o segundas lenguas en entornos virtuales de aprendizaje lleva adscritas una serie de características intrínsecas que hay que tener en cuenta a la hora de desarrollar una plataforma de enseñanza digital. En este trabajo pretendemos mostrar, teniendo presente una experiencia real, cuáles son los elementos claves necesarios para poner en marcha un ambiente virtual destinado a la enseñanza del español como lengua extranjera: la plataforma Eleclips.
\end{abstract}

Palabras clave

Enseñanza de idiomas, entornos virtuales de aprendizaje, español lengua extranjera, Eleclips

\begin{abstract}
Teaching foreign languages or second languages in virtual learning environments has attached a series of intrinsic characteristics that must be considered when developing a digital teaching platform. This paper tries to show, through a real experience, what are the key elements necessary to set up a virtual environment for teaching Spanish as a foreign language: Eleclips platform.
\end{abstract}

Keywords

Language teaching, Virtual learning environments, Spanish foreign language, Eleclips

\section{Introducción}

Los entornos virtuales de aprendizaje (EVA) por su especial configuración exigen una metodología, en cierto modo distante, a la empleada en el aula presencial (Gros Salvat, 2002; Bautista, Borges y Forés, 2006). Podemos hacer referencia a ellos además como AVA (Ambiente Virtual de Aprendizaje) o con sus siglas inglesas VLE (Virtual Learning Environment). Un EVA es:

Un espacio para enseñar y producir aprendizaje, es un ambiente que es modelado pedagógicamente con esa finalidad, donde las diferentes componentes que lo conforman como: los espacios de la plataforma, las actividades y los materiales, buscan generar aprendizaje, el cual se ve enriquecido por la interacción dentro la comunidad de aprendizaje. Dicha interacción es mediada por herramientas informáticas (Quiroz, 2011: 77). 
El desarrollador debe tener presente el carácter de los ambientes virtuales, así como lo que el alumno virtual demanda por sus especiales circunstancias, pero, además, ha de ser consciente de que la propia plataforma o los programas utilizados pueden condicionar el desarrollo y la aplicación didáctica, pues muchas veces no son aptos para realizar las actividades más óptimas o aquellas inicialmente ideadas. Del mismo modo, coordinar a un gran grupo de trabajo conformado por especialistas en la enseñanza del aprendizaje del español como lengua extranjera (ELE) supone ejercer, por un lado, un control estricto que impida la pérdida de coherencia de la plataforma $\mathrm{y}$, por otra parte, la redacción de una guía firme y pautada en la que encuentren unas explicaciones claras y concisas de aquello que tienen que hacer y que permita a los desarrolladores trabajar de la forma más independiente. Son muchos los elementos que entran en juego al enfrentarnos no solo a la creación de contenidos, sino a la implementación digital de los mismos. En esta experiencia salen a la luz los principales problemas que hemos tenido que sortear.

\section{Los entornos virtuales y la enseñanza de idiomas}

Quizá haya sido el aprendizaje de idiomas el último en acceder a los ambientes virtuales. Aunque la educación linguística a distancia ya existía y fue quizá de las pioneras en el aprendizaje fuera del aula, su completa integración en los ámbitos digitales no se ha producido hasta bien tarde. Su penetración en el ámbito educativo es tal que la amplia literatura que existe en torno a su implementación no viene más que a dejar constancia de su importancia (Moore, 1990; Warschauer, 1996; Blake, 2000; Warschauer, 2000, 2002, 2003 y 2006; Dudeney, 2001; Wang y Sun, 2001; Warschauer, Knobel y Stone, 2004; Kern, Ware y Warschauer, 2004; Ware, 2005; White, 2007; Morales Ríos y Ferreira Cabrera, 2008; Jordano de la Torre, 2011).

La llegada de los enfoques comunicativos perjudicó la implantación y desarrollo del aprendizaje de idiomas a distancia, pues, en un primer momento, suponía una dificultad incentivar las destrezas orales sin un interlocutor presencial. No existía más, en los orígenes, que la grabación de un magnetófono, que el estudiante escuchaba y repetía, sin ningún tipo de feedback o interacción. La oralidad quedaba reducida a la prescripción fonética, pero no se daba importancia al uso real y pragmático de toda lengua. Con el desarrollo de las nuevas tecnologías surgieron programas que venían a cubrir este hueco en la enseñanza online. El avance de la técnica permitió que, paulatinamente, la enseñanza de idiomas fuera contando con todas las herramientas e instrumentos necesarios para su desarrollo e implementación (Chapelle, 2003; Chapelle y Douglas, 2006; Blake, 2008). La tecnología ponía a disposición de la enseñanza una serie de recursos que pasarían a denominarse tecnologías de la información y de la comunicación (TIC) y que, sabiamente, muchos supieron utilizar: "hay que aprovechar todo el potencial de las tecnologías de la información y la comunicación (TIC), para orientarse lo básicamente a que todo el alumnado adquiera nuevos y mejores aprendizajes. Este objetivo significa no quedarse en la mera utilización de las tecnologías (TIC), sino aplicarlas en el aprendizaje y el conocimiento (TAC) (Badía, 2009: 3).

La didáctica de la lengua en entornos virtuales de aprendizaje: el caso concreto de la enseñanzaaprendizaje del español como lengua extranjera y la plataforma Eleclips. E. Álvarez Ramos. Página 2 de 20 
Garrison (1993), en un momento temprano, menciona 3 etapas en la enseñanza de lenguas a distancia: correo tradicional, teleconferencia y aprendizaje a través de un ordenador personal. Con la evolución de las nuevas tecnologías podemos hablar, siguiendo a Wang y Sun (2001), de un nuevo peldaño en la enseñanza de idiomas a distancia:

Tabla 1: Etapas de la enseñanza a distancia de lenguas (Wang y Sun, 2001: 541)

Four-Generation Theory

\begin{tabular}{ll}
\hline Generations & Technologies \\
\hline First & Printed media, post system \\
Second & $\begin{array}{l}\text { Older media: printed media, post system } \\
\text { New media: broadcasting, television, radio, telephone, } \\
\text { audiocassettes, videocassettes, cable television, etc. }\end{array}$ \\
Third & $\begin{array}{l}\text { Older media: printed media, post system, broadcast- } \\
\text { ing, television, radio, telephone, audiocassettes, video- } \\
\text { cassettes, cable television, etc. }\end{array}$ \\
& $\begin{array}{l}\text { New media: Word processor, multimedia packages, e- } \\
\text { mail, the Web, the Internet, etc. }\end{array}$ \\
& $\begin{array}{l}\text { Older media: printed media, post system, broadcast- } \\
\text { ing, television, radio, telephone, audiocassettes, video- } \\
\text { Fourth } \\
\text { cassettes, cable television, word processor, multimedia } \\
\text { packages, e-mail, the Web, the Internet, etc. } \\
\text { New media: Internet-based real time technology such } \\
\text { as desktop videoconferencing, Internet telephoning, } \\
\text { virtual reality, etc. }\end{array}$ \\
\hline
\end{tabular}

Dentro de esta evolución podemos hacer referencia a algunos de las páginas en las que podemos encontrar formación en línea de ELE. No todas pueden considerarse como entornos virtuales, pero sí que utilizan los recursos proporcionados por las TIC con una finalidad didáctica. Así, como ya mencionamos en otro lugar (Álvarez Ramos, 2014: 46-47) podemos hacernos eco de: Videoele, un curso de español para extranjeros online y gratuito basado en vídeos (http://www.videoele.com). Los contenidos se organizan en niveles y estructuras gramaticales. La mayoría de las grabaciones carecen de la aplicación didáctica anunciada y no se dispone de solucionarios que nos ayuden en la corrección de los mismos. FluencyProf (https://www.fluencyprof.com) está destinado a anglófonos. Los materiales disponibles son muy variados y disponen de los correspondientes solucionarios que contribuyen a la autonomía del discente. La Familia García Romero (http://www.fundacionlengua.com/garcia-romero/es/componentesfamilia/art/2310/) es es un proyecto desarrollado por la Fundación de la Lengua y la Universidad de Valladolid. Destinado al nivel A1 del Plan Curricular del Instituto Cervantes (PCIC) cuenta con un apartado denominado 'Una escena familiar' donde se explota la comprensión auditiva, también tenemos ejercicios gramaticales, actividades destinadas a la comprensión lectora y aspectos relacionados con el ámbito cultural. El acceso es gratuito, pero es necesario darse de alta en la página. LingusTV (http://www.lingus.tv) es un espacio destinado nuevamente a angloparlantes, en el que la enseñanza se lleva a cabo a través de la explotación didáctica de vídeos. Cada

La didáctica de la lengua en entornos virtuales de aprendizaje: el caso concreto de la enseñanzaaprendizaje del español como lengua extranjera y la plataforma Eleclips. E. Álvarez Ramos. Página 3 de 20 
grabación viene acompañada de una breve nota gramatical con su correspondiente explicación y equivalente en inglés. Mantiene los tradicionales niveles de inicial, intermedio y avanzado.

No todos los EVA desarrollan un aprendizaje semejante, podemos y debemos distinguir entre: e-learning, b-learning; m-learning y u-learning. En el más conocido e-learning la enseñanza-aprendizaje se realiza completamente a distancia, en una plataforma para tal fin a la que se accede vía internet. Se explotan "los medios y dispositivos electrónicos para facilitar el acceso, la evolución y la mejora de la calidad de la educación y la formación” (Sangrá, Vlachopoulos, Cabrera y Bravo, 2011: 36). Los m-learning también representan un ejemplo de la educación a distancia vía internet (Brazuelo, Gallego y Cacheiro, 2017; García-Peñalbo y Ramírez Montoya, 2017), pero en este caso, el rasgo propio radica en hacerlo a través de dispositivos móviles (Sotelo González, 2009; Mora Vicarioli, 2013) que fomenten el aprendizaje (García Aretio, 2017; Ng y Cumming, 2015). Los teléfonos inteligentes posibilitan la instalación de aplicaciones óptimas para el aprendizaje. Su fácil manejo, así como su cómoda portabilidad los han transformado en el primer medio de comunicación (Corbell, 2007; Traxler, 2009; Brazuelo, Gallego y Cacheiro, 2017) y, por ende, en una de las herramientas que más activamente han penetrado en el ámbito de la enseñanzaaprendizaje. El b-learning, sin embargo, combina las sesiones presenciales con la educación a través de un aula virtual (PLS Ramboll Management, 2004; Jenkins, Browne y Walker, 2005). El blended learning permite emplear los aspectos positivos de cada sistema de enseñanza. Se conjuga pues la esencia de los dos tipos de enseñanza: presencial y virtual (Aiello, 2004; Bartolomé Pina, 2004; Wickham, 2014). Se combinan modalidades formativas de trabajo tradicional con sistemas formativos a distancia, se genera así una forma de aprendizaje acorde con las posibilidades actuales de distribución de tiempo, relaciones interpersonales y gestión de espacio (Paniagua, Luengo, Torres Carvalho y Casas, 2017). Para finalizar debemos también mencionar el u-learning, aquel que muestra el carácter ubicuo del aprendizaje que puede llevarse a cabo en cualquier sitio debido a los servicios multiplataforma, las redes móviles y los nuevos medios digitales (Barragán Sánchez, Mimbrero Mallado y Pacheco González, 2013; Velandia-Mesa, Serrano-Pastor y Martínez-Segura, 2017). Se postulan como una de las certidumbres más estables para el futuro en la educación (Jones y Jo, 2004). Todos ellos tienen peculiaridades que les hacen óptimos para el proceso de aprendizaje de lenguas. Será el alumno o el docente el que deba elegir con cuál quiere trabajar.

El hecho de que las tecnologías de la información y la comunicación pongan a disposición del docente una amplia gama de recursos que pueden contribuir al óptimo desarrollo de la enseñanza de un idioma, no trae aparejado que toda docencia en EVA sea aceptable. Todo entorno virtual de aprendizaje ha de cumplir con una serie de requisitos para que, a priori, funcione pedagógicamente (Salinas, 2004). Así, McLoughlin y Oliver (1999) promueven que en todo ambiente virtual hay que conjugar de una manera equilibrada: un marco institucional y organizativo, una tecnología apta y adecuada al fin buscado y un modelo y funciones pedagógicos.

Los EVA aplicados a la enseñanza de idiomas traen consigo un determinado número de ventajas y de inconvenientes que no debemos desdeñar. Entre los beneficios destacamos

La didáctica de la lengua en entornos virtuales de aprendizaje: el caso concreto de la enseñanzaaprendizaje del español como lengua extranjera y la plataforma Eleclips. E. Álvarez Ramos. Página 4 de 20 
los reseñados por Glasserman, Monge y Santiago (2014: 6), para los cuales los entornos virtuales:

- Representan una herramienta de trabajo flexible de interacción mediante actividades sincrónicas y asincrónicas.

- Propician la integración de redes de aprendizaje.

- Facilitan la creación de repositorios de objetos de aprendizaje.

- Desarrollan la autonomía del estudiante y permite la autoevaluación.

- Permiten establecer métodos de enseñanza que promuevan interacción o colaboración entre los participantes.

No debemos olvidar tampoco que los entornos virtuales de aprendizaje representan una clara democratización del acceso a una educación de calidad (García Aretio, 2017; Velandia-Mesa, Serrano-Pastor y Martínez-Segura, 2017).

El alumno puede acceder al aprendizaje en el momento que estime más oportuno y dedicar los minutos que considera más necesarios. No se ve limitado por el tiempo de una clase presencial, tanto por el momento concreto en el que se desarrolla como por la duración de la misma (Borges Sáiz, 2007). Hemos de añadir que, asimismo, puede repetir las actividades las veces que quiera y reincidir en los conceptos en los que tenga más carencias. La autonomía que los EVA conceden al alumno, es, sin duda, el punto fuerte de los mismos, pues priman que el alumno trabaje de forma independiente (García-Peñalbo y Ramírez Montoya, 2017). Esta emancipación del alumno lleva adscrita la necesidad de configuración de los cursos de idiomas en línea, puesto que no existe interacción inmediata con el docente, lo que conlleva una serie de estrategias metodológicas a la hora de plantear y organizar los contenidos y desarrollar la docencia.

Entre las principales desventajas de los EVA hemos de señalar aquellas relacionadas con la soledad del alumno, que no puede interactuar en tiempo real con otros discentes en aquellas plataformas que así lo permiten. Aunque muchas de estas plataformas virtuales tienen habilitadas redes sociales, foros o chats para tal fin o la interacción con el tutor mediante un sistema de tutorías pautadas, la comunicación entre los participantes es quizá el principal asunto pendiente de toda educación a distancia, pues, a pesar de que se pueden habilitar redes sociales, chats o mundos virtuales ad hoc el intercambio comunicativo entre los alumnos dista mucho del experimentado un aula presencial.

Los principales inconvenientes del uso de entornos virtuales tienen que ver directamente con la infraestructura tecnológica. El discente necesita los recursos técnicos necesarios: ordenador, tableta, móvil, etc. y una buena conexión a internet. Asimismo, el docente o del curso deberá adaptar el mismo, al entorno virtual en el que va a desarrollarse y habrá de ajustar su docencia a las funcionalidades del programa implementado o poseer los conocimientos necesarios en el uso y manejo de las TIC para no infrautilizar los recursos que tiene a su alcance (Gros Salvat y Lara Navarra, 2009). Es obligación del profesorado incorporar de forma efectiva las tecnologías para favorecer el aprendizaje (de Benito y Salinas, 2008). Así: "Los procesos didácticos y metodológicos que implican la consecución de tareas 2.0 para el desarrollo de destrezas linguísticas

La didáctica de la lengua en entornos virtuales de aprendizaje: el caso concreto de la enseñanzaaprendizaje del español como lengua extranjera y la plataforma Eleclips. E. Álvarez Ramos. Página 5 de 20 
suponen que la tecnología, al igual que la lengua, es un medio para conseguir el producto final, comunicativo y, en este caso, lingüístico-digital" (Vázquez, 2014: 194).

Al ser la tecnología el principal intermediario en los procesos de enseñanza-aprendizaje, el alumno, que es el principal protagonista del mismo y relega al docente a un segundo plano o incluso lo anula (Valle-López, 2010), también necesita de una serie de habilidades o conocimientos tecnológicos básicos para llevar a cabo el aprendizaje. Beltrán (1996) clasifica estas habilidades necesarias en docentes y discentes y habla de: selección, organización, elaboración, repetición, recuperación, transferencia, metacognición y mediación. Como vemos, la docencia online (García-Aretio, 2017) presenta una singularidad determinada que le imprime un carácter peculiar que debe examinarse para poder alcanzar los objetivos programados. Supone una disrupción educativa que paulatinamente ha ido ganando espacio a la docencia presencial (Ídem), llegando incluso a conseguir resultados de aprendizaje más efectivos que con el uso del tradicional material impreso (Haverila y Barkhi, 2009).

\section{La creación de la plataforma virtual Eleclips}

\subsection{Contexto y objetivos}

En el siguiente apartado queremos presentar de forma descriptiva cuáles han sido las variables que se han tenido en cuenta a la hora de poner en marcha la plataforma virtual Eleclips (http://eleclips.agilicedigital.com), así como cuáles han sido los medios materiales y humanos para llevarla a efecto.

Comenzamos haciendo referencia a las personas involucradas en su gestión y desarrollo. El trabajo ha sido dirigido por el profesor de Lingüística, especializado en la adquisición de segundad lenguas, Robert J. Blake, de University of California, campus de Davis, apoyado por el catedrático de Literatura, Javier Blasco Pascual y por Eva Álvarez Ramos, profesora de Didáctica de la Lengua y la Literatura, ambos de la Universidad de Valladolid. Contamos además con la ayuda en la coordinación académica de Leyre Alejaldre Biel de Mahidol University. International College (Tailandia). Tuvimos a nuestro cargo a un equipo de trabajo formado por egresados en Filología Hispánica y personal en activo dentro del ámbito de ELE.

El proyecto, realizado durante el año 2014, se ejecutó en dos fases una primera etapa de puesta en marcha de la plataforma y desarrollo de contenidos y una segunda etapa de corrección de errores y ampliación de contenidos. Hemos contado con el amparo del spin off Agilice Digital, empresa de base tecnológica (EBT) de la Universidad de Valladolid y que pretende trasferir a la sociedad los resultados de la investigación en distintas ramas de las Humanidades, sobre la base de las TIC. No ha sido subvencionada por ningún programa regional ni estatal.

Dentro del grupo sumábamos más cincuenta años de experiencia en la enseñanza del español como Lengua extranjera y estábamos completamente familiarizados con el mundo de ELE, así como con sus carencias y necesidades. Precisamente de los déficits observados surgió la idea del entorno virtual y quisimos prestar atención tanto a las necesidades de los discentes como a las demandas de los docentes. Entre las principales

La didáctica de la lengua en entornos virtuales de aprendizaje: el caso concreto de la enseñanzaaprendizaje del español como lengua extranjera y la plataforma Eleclips. E. Álvarez Ramos. Página 6 de 20 
carencias del mundo de E/LE nos encontramos con la dificultad de localización precisa y rigurosa de materiales adecuados para un determinado nivel idiomático. La oferta de internet es muy abundante, pero no siempre es fiable o ajustada a los niveles del Plan Curricular del Instituto Cervantes.

Se ideó una estructura orientada al aprendizaje en la que se pudiera trabajar y practicar el idioma. Para ello se adquirió un paquete de grabaciones realizadas por Karen Schairer, profesora de español de la Universidad de Nuevo México, en las que se recogían entrevistas y charlas con diferentes personas de habla hispana originarios de ambos lados del Atlántico. La Dra. Schairer había realizado estas grabaciones con intención de explotarlas didácticamente en sus clases de Español. Accedimos a los materiales en bruto y tuvimos que realizar la correspondiente selección y cortes de los vídeos hasta llegar a configurar un corpus de más de quinientos clips. Después tuvimos que clasificarlos por niveles y adscribir a cada uno un contenido gramatical, uno comunicativo, otro relativo al léxico y, finalmente, otro destinado al componente cultural.

Al estar hablando de aprendizaje de lenguas en entornos virtuales teníamos que considerar dos grandes aspectos como son los lingüísticos y los tecnológicos. Los contenidos a desarrollar debían tener la misma importancia y generarse de igual modo que si de una clase presencial se tratara. El uso de nuevas tecnologías no debe enmascarar contenidos vacuos o de poca calidad. Ambos elementos han de ser tratados con el mismo rigor. El fin último es la consecución del objetivo educativo (Zapata-Ros, 2015; García-Peñalbo y Ramírez Montoya, 2017). Del mismo modo, es necesario adecuarse a los nuevos roles adquiridos por profesores y alumnos en los entornos virtuales de aprendizaje ((Lynch, 2002; Majó y Marquès, 2002; McLoughlin y Lee, 2008; Romeu, 2011). Los cambios derivados de la implementación de las TIC en educación inciden directamente en dichos procesos de enseñanza-aprendizaje (ZapataRos, 2010; Álvarez Acosta, Avello Martínez y López Fernández, 2012).

También nos pareció oportuno que los alumnos tuvieran a su disposición toda la amplia gama de variedades diatópicas del castellano, que la configuran como una lengua rica y en constante progreso unida a una red intercultural de vital importancia en la enseñanzaaprendizaje de una lengua. Es principalmente esta pluralidad de hablas y variedades culturales lo que confieren al español su verdadero carácter. Consideramos que era necesario proporcionar al alumno muestras de hablas reales tan apartadas de los audios enlatados de los cursos tradicionales de idiomas. Así pues, cuatro fueron los aspectos lingüísticos principales:

- Ajustarnos a los niveles del Plan curricular del Instituto Cervantes

- Proporcionar todas las variedades diatópicas de la lengua española

- Ofrecer muestras reales de habla.

- Mostrar los elementos culturales propios adscritos a cada una de estas variedades lingüísticas.

Convertimos, pues, las carencias en estos cuatro ejes principales sobre los que asentar la plataforma. Eleclips es el resultado de una fórmula que integra cuatro elementos fundamentales para el trabajo y aprendizaje de español:

La didáctica de la lengua en entornos virtuales de aprendizaje: el caso concreto de la enseñanzaaprendizaje del español como lengua extranjera y la plataforma Eleclips. E. Álvarez Ramos. Página 7 de 20 
1. aprovecha al máximo las nuevas tecnologías;

2. parte de un fondo audiovisual singular;

3. cuenta con el respaldo de un equipo de especialistas en la enseñanza de español como segunda lengua, que trabaja con rigor y que está avalado por años de experiencia en las aulas;

4. se preocupa por mostrar todas las variantes diatópicas y culturales del castellano siguiendo los parámetros marcados en el Plan curricular del Instituto Cervantes.

\subsection{Método y enfoque pedagógico}

Queríamos darle un preeminente enfoque comunicativo siguiendo las tendencias pedagógicas actuales más extendidas en la enseñanza de lenguas, aquellas que apoyan un modelo lingüístico activo y comunicativo (Contreras Izquierdo, 2008). Apostamos por la oralidad como elemento principal de la plataforma. Con ello se pretende capacitar al estudiante para una comunicación real, de ahí que sea expuesto a materiales reales y grabaciones auténticas. Las actividades gramaticales derivadas de las mismas procuran imitar la realidad comunicativa (Brumfit, 1984; Candlin, 1981; Sánchez, 1997; Melero, 2000; Cortés Moreno, 2000).

La enseñanza de lenguas se concibe no solo como la adquisición de ciertas competencias gramaticales, sino que los componentes sociocultural y pragmático son elementos indispensables en dichos procesos. En la cultura subyacente a la comunicación participan elementos relacionados directamente con los actos de habla, propios de la competencia pragmática, sociolingüística y sociocultural: "cuestiones extralingüísticas como la gestualización o la proxémica [...] revisten una importancia crucial en el correcto devenir de la conversación. ¿Y qué decir de otros aspectos socioculturales tan distintos entre las diferentes lenguas como el sentido del humor, la jerarquía social y sexual, los hábitos alimenticios, gustos y etiqueta social, etc." (Mateo Martínez, 1999: 172). El fin último es conseguir que el alumno adquiera competencias que le conviertan en hablante intercultural (Mody, 2002; O’Dowd, 2003).

Se fomenta el desarrollo de todas las destrezas orales y escritas a excepción de la expresión oral que, por las limitaciones del programa elegido, eXeLearning (http://exelearning.net), ha de ser trabajada mediante charlas con el profesor-tutor asignado. Las actividades se distribuyen en 4 niveles: gramática, funciones comunicativas, léxico y conocimientos culturales. Aspectos que se trabajan con la variedad de ejercicios que proporciona programa empleado.

Utilizamos un corpus de vídeos de habla natural y espontánea, los audios recogen errores inherentes a la oralidad: frases inconclusas, sinalefa de elementos en la cadena hablada; apócopes, metátesis, elipsis de alguna palabra, interrupciones, solapamientos en los discursos... El estudiante es consciente en todo momento de que se enfrenta a un uso real de la lengua y hay que advertirle de que, son, precisamente esos errores los que le imprimen el carácter propio a la lengua hablada. El discente ha de ser consciente de cuál es el habla real que se utiliza fuera del aula. Los discursos orales, que tradicionalmente se utilizan en el mundo de ELE para ejercitar la competencia auditiva (CA), adolecen de cierta artificialidad, el ritmo discursivo es lento y uniforme; la

La didáctica de la lengua en entornos virtuales de aprendizaje: el caso concreto de la enseñanzaaprendizaje del español como lengua extranjera y la plataforma Eleclips. E. Álvarez Ramos. Página 8 de 20 
entonación se desarrolla con oscilaciones exageradas; el vocabulario está controlado según el nivel de los alumnos; las oraciones completas (nunca fraccionadas e inconclusas) se adecuan al nivel gramatical estudiado y se repiten determinadas formas linguiísticas. Si a esto le unimos la querencia a la ralentización del discurso, la ausencia, casi total, de inferencias y el perfeccionismo gramatical cercano al academicismo (nunca encontraremos en una audición un error de habla), nos encontramos con unos materiales, que poco o nada tienen que ver con la rica y auténtica habla natural real. Las audiciones intentan imitar el lenguaje oral y sus rasgos suprasegmentales, pero, aunque se intente dotarlas de naturalidad, toda actividad destinada a ejercitar esta destreza comunicativa no deja de ser una impostura. (Álvarez Ramos y Blasco, 2015; Álvarez Ramos, 2014): "La comprensión auditiva no puede basarse en la escucha repetida de ciertas estructuras modelos porque en la vida real al alumno se le van a exigir otro tipo de capacidades, tendrá que cubrir otras necesidades" (Álvarez Ramos, 2014: 42).

Además, es muy común que se tienda a usar un registro estándar del lenguaje, que normalmente coincide con el español peninsular. La opción fue emplear grabaciones realizadas en todos y cada uno de los países de habla hispana, de ahí que se recojan todos los acentos del castellano que hacen de ella una lengua viva, fértil y nutrida. Hasta la fecha parece ser que las enseñanzas del español venían pautadas por la norma establecida en la península ibérica que marcaba cuál era la lengua estándar en un reduccionismo lingüístico y cultural bastante significativo.
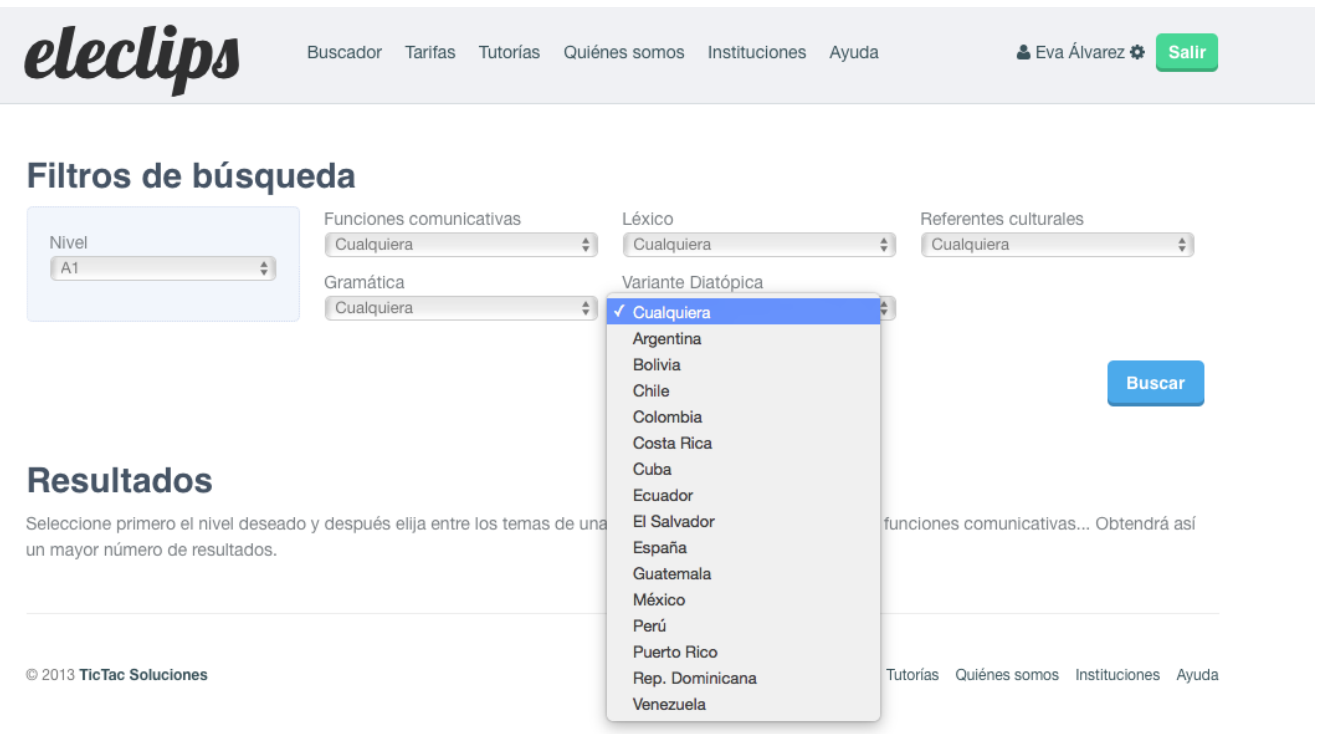

Imagen 1: Muestra de variedades del español presentes en la plataforma Eleclips

\subsection{Procedimiento y desarrollo}

Es cierto que, en la era de la comunicación, el docente y el discente tienen a mano en internet multitud de recursos, aunque han de preguntarse, si esos recursos siguen las pautas lingüísticas dadas por el Instituto Cervantes en su Plan Curricular; si esos materiales cumplen realmente el objetivo de enseñar y si son capaces de explotar todas las herramientas que brinda internet (Piñol, 2004). La red está plagada de materiales para aprender cualquier lengua, bien de particulares que comparten sus materiales bien de empresas que gestionan este aprendizaje (una búsqueda en Google sobre cursos de

La didáctica de la lengua en entornos virtuales de aprendizaje: el caso concreto de la enseñanzaaprendizaje del español como lengua extranjera y la plataforma Eleclips. E. Álvarez Ramos. Página 9 de 20 
español para extranjeros devuelve más de 35 millones de páginas). El problema surge cuando hay que seleccionar entre tanta oferta cuáles son las de mayor calidad, porque tal y como señala Mar Cruz Piñol (2002), hay que distinguir entre navegar en la red y naufragar en la misma.

Creímos necesario ajustar nuestra plataforma a lo pautado en el PCIC. Se cumplieron escrupulosamente todas las indicaciones dadas en el mismo. En la nivelación se han tenido en cuenta las directrices que marca el Plan curricular, así como aspectos lingüísticos (los relativos a la gramática, frases hechas...) y extralingüísticos (velocidad y claridad de dicción, ruidos de fondo...). Este no es un hecho baladí, pues existe toda una amplia gama de materiales que lucen el distintivo de adaptación al Plan Curricular y lo que han hecho, simplemente, ha sido dividir los antiguos niveles Inicial, Intermedio, Avanzado y Superior, en subniveles poniéndoles la etiqueta de A1, A2 ... No se ha producido, por tanto, dicha adaptación más que en el cambio de nomenclatura. Es un hecho del que somos conocedores todos los docentes e investigadores dedicados al mundo de ELE o a la enseñanza de segundas lenguas, pues ha sido una práctica general de gran parte de la editoriales y portales de enseñanza.

Nos pareció óptimo darle un papel principal al fomento de la competencia auditiva y gestionar la plataforma en torno a los recursos audiovisuales. Recursos que por otra parte nos ofrecía el entorno digital. El alumno podía trabajar aspectos gramaticales, comunicativos, léxicos y culturales a través del visionado de vídeos. La gestión de los materiales comprendió varios pasos: primero, transcribimos las locuciones; luego, catalogamos, clasificamos y etiquetamos los vídeos siguiendo las directrices establecidas en el PCIC, para determinar en cada uno de ellos aquellos campos en los que mejor podían aprovecharse sus contenidos: nivel de español, gramática, léxico, funciones comunicativas, referentes culturales; finalmente, elaboramos ejercicios y actividades para aprovechar las posibilidades de cada uno y poder trabajar tanto la comprensión auditiva y lectora, como la expresión oral y escrita, o practicar, además, la gramática, el léxico y las funciones comunicativas. La mermada producción oral, derivada de las limitaciones del programa empleado, se ve suplida mediante la introducción de un profesor tutor del lenguaje oral, con el que se puede charlas vía Skype. Ha sido quizá este uno de los hándicaps adscritos a los entornos virtuales destinados a la enseñanza de idiomas. La comunicación entre el profesor y el alumno podía resultar, a veces, inexistente, pero en la actualidad gracias a los avances tecnológicos esta distancia parece haberse solventado (Holmberg, 1995; Moore y Cozine, 2000). Elegimos la plataforma anteriormente señalada puesto que nos permitía realizar conversaciones cara a cara, hecho que permite la visualización de los gestos faciales, tan vitales en el desarrollo de la oralidad (Jordano de la Torre, 2011).

Los aspectos tecnológicos ocuparon, como ya hemos dicho, una posición principal en el desarrollo e implementación de la plataforma. La interfaz de la misma es bastante sencilla y muy intuitiva. Queríamos que el usuario se familiarizara rápidamente con su funcionamiento y nos ajustamos a los parámetros derivados de la "usabilidad" digital promovidos por Nielsen (1999). Siguiendo estas máximas, Pérez Vicente (2012) especifica que la usabilidad se basa en cinco elementos:

La didáctica de la lengua en entornos virtuales de aprendizaje: el caso concreto de la enseñanzaaprendizaje del español como lengua extranjera y la plataforma Eleclips. E. Álvarez Ramos. Página 10 de 20 
1. Facilidad de aprendizaje: hasta qué punto es fácil para los usuarios realizar las tareas básicas cuando se enfrentan por primera vez a un diseño.

2. Eficiencia: una vez que los usuarios ya han aprendido el diseño, con qué rapidez realizan las tareas.

3. Facilidad para ser recordado: cuando los usuarios han dejado durante un tiempo de interactuar con ese diseño, cuánto les cuesta recordar cómo se utilizaba.

4. Errores: cuántos errores cometen los usuarios al enfrentarse con el diseño, por qué, son de gravedad, los pueden solventar fácilmente.

5. Satisfacción: ¿es agradable utilizar el diseño?, ¿son fácilmente localizables los elementos en él?, ¿ayuda o dificulta la realización de las tareas?, ¿volverían los usuarios a utilizar de nuevo ese diseño? (71-72)

Ideamos un buscador en el que todos los vídeos pudieran ser consultados y recuperados a partir de varios parámetros: contenidos gramaticales, funciones comunicativas, léxico, conocimientos culturales, variedad diastrática y niveles de dificultad siguiendo los parámetros del Marco Común Europeo de Referencia para las lenguas (MCER) y respetando los contenidos del PCIC.

En función de la elección realizada, la herramienta devuelve todas las opciones que cumplen esos parámetros. Y, entre las alternativas resultantes, se puede elegir la que desee con la seguridad de que tanto el clip como las actividades que lo acompañan se corresponderán con su elección de nivel de español, de gramática, de léxico, de funciones comunicativas y de referente cultural. La oferta es muy variada respondiendo a la demanda del alumno virtual que como consumidor y prosumidor de contenidos digitales, exige esa pluralidad de respuestas a su búsqueda.

\section{Filtros de búsqueda}
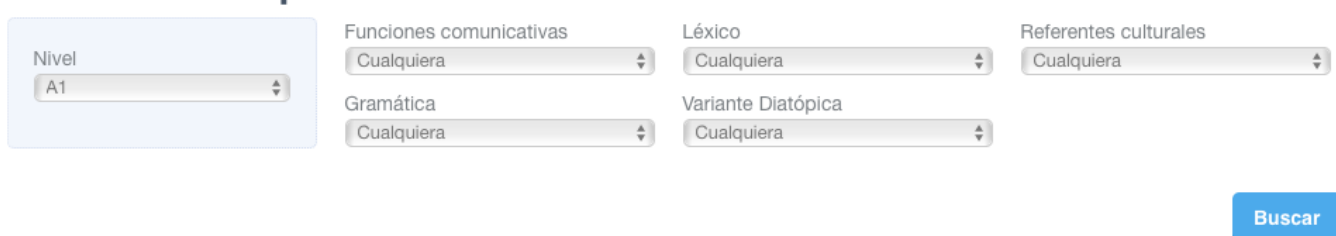

\section{Resultados}

Imagen 2: muestra del buscador de contenidos del EVA Eleclips

Es vital en todo EVA que el usuario sea capaz de encontrar todo rápidamente y que la organización, estructura y funcionamiento de la página sea un aliado y no un enemigo. La mala resolución y ubicación de los elementos principales de un entorno de aprendizaje conlleva aparejada cierta frustración o negatividad que afecta directamente al aprendizaje. Las premisas de la neurodidáctica apuntan a que los ambientes cobran importancia en los procesos de adquisición del conocimiento, pues repercuten directamente en la motivación (Robinson, 2016; Mora, 2013).

La tipología de los ejercicios viene pautada por el programa básico empleado para las actividades didácticas. Optamos por aprovechar las posibilidades que nos daba

La didáctica de la lengua en entornos virtuales de aprendizaje: el caso concreto de la enseñanzaaprendizaje del español como lengua extranjera y la plataforma Eleclips. E. Álvarez Ramos. Página 11 de 20 
eXeLearning por ser una herramienta gratuita que puede ser usada en muchos dispositivos y que facilita la transformación a diferentes formatos como e-pub, SCORM o PDF. Tal y como puede leerse en la página de eXeLearning: es un programa libre y abierto bajo licencia GPL-2, para ayudar a los docentes en la creación y publicación de contenidos, y que permite a profesores y académicos la publicación de contenidos didácticos en soportes informáticos ( $\mathrm{CD}$, memorias USB, en la web), sin necesidad de ser ni convertirse en expertos en HTML, XML o HTML5; y disponible en GNU/Linux, Microsoft Windows y Mac OS X. Los recursos creados en eXelearning son accesibles en formato XHTML o HTML5, pudiendo generarse sitios web completos (páginas web navegables), insertar contenidos interactivos (preguntas y actividades de diferentes tipos) en cada página, exportar los contenidos creados en otros formatos como ePub3 (un estándar abierto para libros electrónicos), IMS o SCORM (estándares educativos que permiten incorporar los contenidos en herramientas como Moodle), XLIFF (un estándar para la traducción) y catalogar los contenidos con diferentes modelos de metadatos: Dublin Core, LOM, LOM-ES. Permite, en resumen, vadear las lagunas o carencias tecnológicas del docente, facilitándole el autoabastecimiento de recursos digitales. Se sacrifica la vistosidad por la utilidad, su fácil manejo y su óptima implementación.

? Actividad desplegable

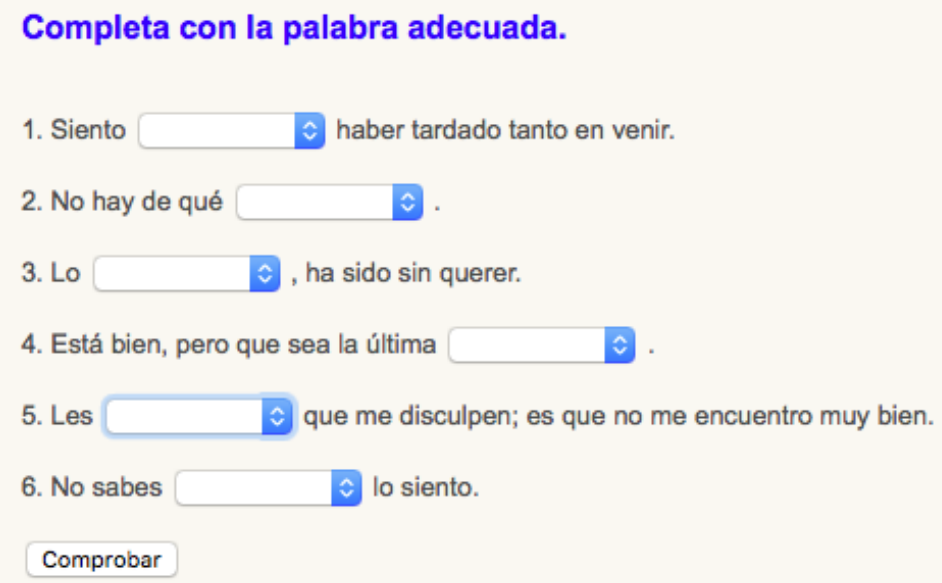

Imagen 3: Ejemplo de actividad de eXelearning

Las limitaciones con las que nos hemos encontrado en la plataforma Eleclips son las derivadas del formato del programa empleado, el reducido número de tipologías de ejercitación y quizá el aspecto no excesivamente atractivo que presentan las actividades, de un formato un tanto plano, pero cumplen con las necesidades didácticas requeridas. Todos los ejercicios son autocorregibles y cuando el discente termina de realizarlos puede comprobar in situ y al momento los errores y aciertos cometidos. Ha primado la utilidad por encima de la estética.

Configurar un ambiente de aprendizaje de lenguas con enfoque comunicativo conlleva además concederle una mayor importancia al desarrollo de la oralidad sobre todo en su nivel expresivo. En nuestro caso esta necesidad se cubre, solamente, desde la presencia

La didáctica de la lengua en entornos virtuales de aprendizaje: el caso concreto de la enseñanzaaprendizaje del español como lengua extranjera y la plataforma Eleclips. E. Álvarez Ramos. Página 12 de 20 
de charlas con el tutor asignado. Este es otro de los puntos más débiles de la plataforma en particular y del resto de ambientes virtuales destinados a la enseñanza de lenguas en general.

\section{Para concluir}

Llevar a cabo este proyecto ha requerido de esfuerzo y tiempo y en muchas ocasiones nos hemos tenido que dejar llevar por el método de ensayo y error, puesto que se trataba de nuestra primera incursión en el medio digital. Se ha intentado en todo momento darle valor y calidad al contenido y hacer un buen uso de las tecnologías poniéndolas al servicio de la educación y no a la inversa. De ahí que estemos ante una herramienta que, utilizando el lenguaje natural, es un excelente campo de entrenamiento y ejercitación para que el estudiante practique y aprenda por su cuenta, ya que cada ejercicio se autocorrige y se acompaña de la información pertinente para que el discente pueda aprender resolviendo problemas. La tecnología puede ser una herramienta muy productiva para el aprendizaje y la enseñanza de idiomas, si bien esta ha de usarse de manera correcta, promoviendo la reflexión y reforzando la resolución de problemas (Murray, 1999). Los alumnos se sienten, además, confiados al poder trabajar con materiales reales y no con sucedáneos de la rica lengua natural. El trabajo en entornos virtuales con la lengua real prepara al discente para su contacto con el mundo y lo aleja de las fingidas, reiterativas y edulcoradas conversaciones de los manuales al uso.

Pero también puede ser destinada a facilitar el trabajo del docente, que encuentra todo el material que necesita para su clase rápidamente, puesto que los contenidos se han estructurado y se han realizado pensando en desarrollar una herramienta para facilitar la labor en clase al profesor y para que alumnos independientes aprendan online. Estaríamos pues hablando de una enseñanza en la que se asimilarían todos los aprendizajes señalados anteriormente: e-learning, b-learning; m-learning. Puede además realizarse como curso teniendo presentes los niveles del PCIC. Las búsquedas cruzadas nos ofrecen los resultados más óptimos. Además, se promueve la autonomía del alumno al ser el responsable del camino de su aprendizaje, eligiendo con total libertad los contenidos que quiere tratar o aquellos que ha de reforzar. No debemos olvidar que, al ser una plataforma en línea, no hay horarios y cada usuario elige cuándo comenzar y dejar el aprendizaje. Y como los ejercicios se corrigen automáticamente, no se necesita de la presencia de un profesor o tutor que gestione el aprendizaje. Es precisamente este uno de los criterios principales que el desarrollador ha de tener en cuenta para alcanzar el logro del aprendizaje. Es de vital necesidad "potenciar el tránsito progresivo de la dependencia a la independencia y a la autorregulación" (Álvarez Acosta, Avello Martínez y López Fernández 2012: 2).

El docente no es sustituido al $100 \%$ con este sistema de aprendizaje, pues sigue siendo él el gestor de los contenidos. Ya UNESCO, en su informe mundial sobre educación, mantenía la presencia del docente en los entornos virtuales, así: "la nueva tecnología de la información no hace que los docentes dejen de ser indispensables, sino que modifica su papel en relación con el proceso de aprendizaje, y que el diálogo permanente que transforma la información en conocimiento y comprensión pasa a ser fundamental" (s. p.). Su localización virtual y su digitalización permiten que el alumno pueda trabajar

La didáctica de la lengua en entornos virtuales de aprendizaje: el caso concreto de la enseñanzaaprendizaje del español como lengua extranjera y la plataforma Eleclips. E. Álvarez Ramos. Página 13 de 20 
tanto dentro como fuera de la clase. En esta doble perspectiva de la plataforma Eleclips, las aulas también pueden seguir ocupando un lugar privilegiado, pues permite que sea empleada como una actividad más.

Se deja abierta a la elección de los usuarios su utilidad como un elemento más en el aula o su uso como actividad individual. Hemos querido realizar un entorno virtual de aprendizaje apto y óptimo para todos.

Presentación del artículo: 22 de abril de 2017

Fecha de aprobación: 9 de diciembre de 2017

Fecha de publicación: 22 de diciembre de 2017

Álvarez Ramos, E. (2017). La didáctica de la lengua en entornos virtuales de aprendizaje: el caso concreto de la enseñanza-aprendizaje del español como lengua extranjera y la plataforma Eleclips. RED. Revista de Educación a Distancia, 55. Consultado el (dd/mm/aaaa) en http://www.um.es/ead/red/55/alvarez.pdf

\section{Financiación}

Esta investigación no ha recibido ninguna subvención específica de los organismos de financiación en los sectores públicos, comerciales o sin fines de lucro.

\section{Referencias bibliográficas}

Aiello, M. (2004). El blended learning como práctica transformadora. Pixel-Bit: Revista de medios y educación, 23, 21-26.

Álvarez Acosta, H., Avello Martínez, R., y López Fernández, C. R. (2013). Los entornos virtuales de aprendizaje como recurso didáctico en el ámbito universitario. Universidad y Sociedad, 5 (1), 1-10.

Álvarez Ramos, E. (2014). Internet, Biblioteca sonora: análisis, tratamiento e integración de recursos auditivos para el aula de ELE. Didáctica. Lengua y Literatura, 26, 39-55.

Álvarez Ramos, E., y Blasco Pascual, J. (2015). Eleclips, un recurso a medida en la enseñanza aprendizaje de ELE: de los fingidos actos de habla tradicionales a la autenticidad de las hablas reales. En M. P. Celma Valero, S. Heikel y C. Morán Rodríguez (Eds.), Portugal y España, tierras de encuentro y proyección cultural. Coloquio Internacional de Aepe. Valladolid: Asociación Europea de profesores de Español, 53-62.

Badía, J. (2009). Presentación. En R. Fronell y J. Vivancos. El plan TAC del centro. Generalitat de Catalunya: Departamento de educación, 3.

La didáctica de la lengua en entornos virtuales de aprendizaje: el caso concreto de la enseñanzaaprendizaje del español como lengua extranjera y la plataforma Eleclips. E. Álvarez Ramos. Página 14 de 20 
Barragán Sánchez, R., Mimbrero Mallado, C., y Pacheco González Piñal, R. (2013). Cambios pedagógicos y sociales en el uso de las TIC: u-learning y u-portafolio. Revista Electrónica de Investigación y Docencia, 10, 7-20.

Bartolomé Pina, A. R. (2004). Blended learning: conceptos básicos. Pixel-Bit: Revista de medios y educación, 23, 7-20.

Bautista, G., Borges, F., y Forés, A. (2006). Didáctica universitaria en entornos virtuales de enseñanza- aprendizaje. Madrid: Narcea.

Beltrán, J. (1996). Procesos cognitivos y soportes tecnológicos. En F. J. Tejedor y A. G. Valcárcel (Eds.), Perspectivas de las nuevas tecnologías en la educación. Madrid: Narcea, 63-86.

Blake, R. J. (2000). Computer mediated communication: A window on L2 Spanish interlanguage. Language, Learning \& Technology, 4 (1), 120-136.

Blake, R. J. (2008). Brave New Digital Classroom: Technology and Foreign Language Learning. Washington, DC: Georgetown University Press.

Borges Sáiz, F. (2007). El estudiante de entornos virtuales. Una primera aproximación. Digithum, 9, 1-7.

Brazuelo Grund, F., Gallego Gil, D. J., y Cacheiro González, M. L. (2017). Los docentes ante la integración educativa del teléfono móvil en el aula. RED Revista de Educación a Distancia, 56, 1-22. DOI: http://dx.doi.org/10.6018/red/52/6.

Brumfit, C. (1984). Communicative Methodology in Language Teaching: the Roles of Fluency and Accuracy. Cambridge: C.U.P.

Candlin, C. N. (ed.), (1981). The Communicative Teaching of English: Principles and Exercise Typology. Londres: Longman

Chapelle, C. A. (2003). English language learning and technology: Lectures on applied linguistics in the age of information and communication technology. Amsterdam: John Benjamins.

Chapelle, C. A., y Douglas, D. (2006). Assessing language through computer technology. Cambridge: Cambridge University Press.

Contreras Izquierdo, N. M. (2008). La enseñanza-aprendizaje de lenguas extranjeras y las TICs: el caso del Español como Lengua Extranjera (ELE). Iniciación a la investigación, 3 (4), I-VII.

Corbell, J. (2007). Are you ready for mobile learning? EDUCAUSE Quarterly, 30 (2), 51-58.

La didáctica de la lengua en entornos virtuales de aprendizaje: el caso concreto de la enseñanzaaprendizaje del español como lengua extranjera y la plataforma Eleclips. E. Álvarez Ramos. Página 15 de 20 
Cortés Moreno, M. (2000). Guía para el profesor de idiomas: didáctica del español y segundas lenguas. Barcelona: Octaedro.

De Benito, B., y Salinas, J. (2008). Los entornos tecnológicos en la Universidad. PíxelBit. Revista de Medios y Educación, 32, 83-100.

Dudeney, G. (2001). The internet and the language classroom: A practical guide for teachers. Cambridge: Cambridge University Press.

García-Aretio, L. (2017). Educación a distancia y virtual: calidad, disrupción, aprendizajes adaptativo y móvil. RIED. Revista Iberoamericana de Educación a Distancia, 20 (2), 9-25. DOI: http://dx.doi.org/10.5944/ried.20.2.18737.

García-Peñalvo, F. J., y Ramírez Montoya, M.S. (2017). Aprendizaje, Innovación y Competitividad: La Sociedad del Aprendizaje. RED. Revista de Educación a Distancia, 52, 1-6. DOI: http://dx.doi.org/10.6018/red/52/1.

Garrison, D. R. (1993). Quality and access in distance education. En: Keegan, D. (ed), Theoretical principles of distance education (9-21). London: Routledge.

Glasserman, L. D., Monge, P., y Santiago, J. M. (2014). Experiencia de enseñanzaaprendizaje con la plataforma educativa abierta Moodle. En Memoria del Congreso Iberoamericano de Ciencia, Tecnología, Innovación y Educación. Buenos Aires: Organización de Estados iberoamericanos para la Educación la Ciencia y la Cultura, 2-10.

Gros Salvat, B. (2002). Constructivismo y Diseño de Entornos Virtuales de Aprendizaje. RE. Revista de Educación. Didácticas Específicas, 1, 225- 250.

Gros Salvat, B. y Lara Navarra, P. (2009). Estrategias de innovación en la educación superior: el caso de la Universidad Oberta de Catanlunya. Revista Iberoamericana de Educación, 49, 223-245.

Haverila, M., y Barkhi, R. (2009). The influence of experience, ability and interest on eLearning effectiveness. European Journal of Open, Distance and E-Learning, 1 , s. p. (Consultado 2 de diciembre de 2017). Disponible en: http://www.eurodl.org/materials/contrib/2009/Haverila_Barkhi.htm

Holmberg, B. (1995). Theory and practice of distance education. London: Routledge.

Instituto Cervantes (2006). Plan curricular del Instituto Cervantes. Niveles de referencia para el español. Madrid: Instituto Cervantes- Biblioteca nueva. Disponible en: http://cvc.cervantes.es/ensenanza/biblioteca_ele/plan_curricular/

Jenkins, M., Browne, T. y Walker, R. (2005). VLE Surveys. A longitudinal perspective

La didáctica de la lengua en entornos virtuales de aprendizaje: el caso concreto de la enseñanzaaprendizaje del español como lengua extranjera y la plataforma Eleclips. E. Álvarez Ramos. Página 16 de 20 
between March 2001, March 2003 and March 2005 for higher education in the United Kingdom. Oxford: UCISA-Universidad de Oxford.

Jones, V., y Jo, J. H. (2004). Ubiquitous learning environment: An adaptive teaching system using ubiquitous technology. En R. Atkinson, C. McBeath, D. JonasDwyer y R. Phillips (eds.), Beyond the comfort zone: Proceedings of the 21st ASCILITE Conference, (468-474). Perth, 5-8 de diciembre. (Consultado 4 de diciembre de 2017). Disponible en: https://www.ascilite.org/conferences/perth04/procs/jones.html

Jordano de la Torre, M. (2011). La enseñanza-aprendizaje de la competencia oral en lengua extranjera en el contexto de la educación abierta y a distancia: de la casete a la interacción virtual. RIED. Revista Iberoamericana de Educación a Distancia, 14 (1), 15- 39. DOI: https://doi.org/10.5944/ried.1.14.798

Kern, R., Ware, P., y Warschauer, M. (2004). Crossing frontiers: New directions in online pedagogy and research. Annual Review of Applied Linguistics, 24, 243260.

Lynch, M. (2002). The online educator. A guide to creating the virtual classroom. Nueva York: RoutledgeFalmer.

Majó, J., y Marquès, P. (2002). La revolución educativa en la era internet. Barcelona: CissPraxis.

Mateo Martínez, J. (1999). La enseñanza universitaria de las lenguas extranjera. Alicante: Universidad de Alicante Publicaciones.

McLoughlin, C., y Oliver, R. (1999). Pedagogic roles and dynamics in telematics environments. En M. Selinger y J. Pearson (eds.), Telematics in Education: trends and issues (32-50). Oxford: Elsevier Science.

McLoughlin, C., y Lee, M. (2008). Mapping the digital terrain: New media and social software ascatalysts for pedagogical change. En A. Farley y D. Holt, Hello! Where are you in the landscape of educational technology? (pp. 641-652). Melbourne: Deakin University.

Melero, P. (2000). Métodos y enfoques en la enseñanzalaprendizaje del español como lengua extranjera. Madrid: Edelsa.

Mody, W. (2002). Handbook of International and Intercultural Communication. California: SAGE Publications.

Mora, F. (2013). Neuroeducación. Madrid: Alianza.

Mora Vicarioli, F. (2013). El mobile learning y algunos de sus beneficios. Calidad en la Educación Superior, 4-1, 47-67.

La didáctica de la lengua en entornos virtuales de aprendizaje: el caso concreto de la enseñanzaaprendizaje del español como lengua extranjera y la plataforma Eleclips. E. Álvarez Ramos. Página 17 de 20 
Morales Ríos, S., y Ferreira Cabrera, A. (2008). La efectividad de un modelo de aprendizaje combinado para la enseñanza del inglés como lengua extranjera: estudio empírico. RLA. Revista de Lingüística Teórica y Aplicada, 46 (2), 95118.

Moore, M. G. (1990). Recent contributions to the theory of distance education. Open Learning: The Journal of Open and Distance Learning, 5 (3), 10-15.

Moore, M. G., y Cozine, G. T. (Eds.). (2000). Web-based communications, the Internet and distance education. University Park, PA: American Center for the Study of Distance Education.

Murray, T. (1999). Authoring Intelligent Tutoring Systems: An Analysis of the State of the Art. International Journal of Artificial Intelligence in Education 10, 98-129.

Nielsen, J. (1999). Designing Web Usability: The Practice of Simplicity. New Riders Publishing Thousand Oaks: California.

Ng, W., y Cumming, T. M. (Eds.) (2015). Sustaining Mobile Learning: Theory, Research and Practice. Londres: Routledge.

O'Dowd, R. (2003). Understanding the "other side": Intercultural learning in a SpanishEnglish email exchange. Language Learning \& Technology, 7 (2), 118-144.

Paniagua, A., Luengo, R., Torres Carvalho, J.L., y Casas, L.M. (2017). Blended learning en la formación permanente del profesorado. RED. Revista de Educación a Distancia, 52, 1-15. DOI: http://dx.doi.org/10.6018/red/52/3

Pérez Vicente, A. (2012). Usabilidad. Qué debo tener en cuenta al escribir para la web. Escribir para los medios. En M. Tascón (dir.), Guía para los nuevos medios y las redes sociales. Barcelona: Círculo de lectores, 71-72.

Piñol, M. C. (2002). ¿Navegar o naufragar? La WWW y la enseñanza del léxico del español. Textos. Didáctica de la lengua y la literatura, 31, 53-64

Piñol, M. C. (2004). La enseñanza de léxico en el aula de español como lengua extranjera. Recursos en Internet para la elaboración de actividades. Carabela, $56,171-77$

PLS Ramboll Management (2004). Studies in the Context of the E-learning Initiative: Virtual Models of European Universities (Lot). Draft Final Report to the EU Commission, DG Education \& Culture. EU Commission, DG Education y

Quiroz, J. S. (2011). Diseño y moderación de entornos virtuales de aprendizaje (EVA): Editorial UOC, S.L.

La didáctica de la lengua en entornos virtuales de aprendizaje: el caso concreto de la enseñanzaaprendizaje del español como lengua extranjera y la plataforma Eleclips. E. Álvarez Ramos. Página 18 de 20 
Robinson, K. (2016). Escuelas creativas. Barcelona: Debolsillo.

Romeu, T. (2011). La docencia en colaboración en contextos virtuales. Estudio de caso de un equipo de docentes del área de competencias digitales de la UOC. Tesis doctoral. UOC. (Consultada 4 de diciembre de 2017). Disponible en: openaccess.uoc.edu/webapps/o2/bitstream/10609/18101/.../Tesi_Teresa_Romeu _v2.pdf

Salinas, J. (2004). Cambios metodológicos con las TIC. Estrategias didácticas y entornos virtuales de enseñanza-aprendizaje. Bordón. Revista de pedagogía, 56, 3-4, 469-481.

Sánchez, A. (1997). Los métodos en la enseñanza de idiomas. Evolución histórica y análisis didáctico. Madrid: SGEL.

Sangrà, A., Vlachopoulus, D, Cabrera, N., y Bravo, S. (2011). Hacia una definición inclusiva del e-learning. Barcelona: eLearn Center. UOC. (Consultado 2 de diciembre de 2017). Disponible en: http://openaccess.uoc.edu/webapps/o2/bitstream/10609/10541/6/inf_ed_cast.pdf

Sotelo González, J. (2009). Del "e-learning” al "m-learning": una academia en cada "iPhone". Telos: Cuadernos de comunicación e innovación, 82, 122-128.

Traxler, J. (2009). Learning in the Mobile Age. International Journal of Mobile and Blended Learning, 1 (1), 1-12. (Consultado 7 de diciembre de 2017). Disponible en: https://www.academia.edu/171500/Learning_in_a_Mobile_Age

UNESCO (1999). Declaración mundial sobre la educación superior en el siglo XXI: visión y acción. s. p. Aprobada por la Conferencia Mundial Sobre Educación Superior, realizada en París en 1998. (Consultado 23 de marzo de 2017) Disponible en: http://unesdoc.unesco.org/images/0011/001163/116345s.pdf

Valle-López, J. M. (2010). El Proceso de Bolonia: un nuevo marco de aprendizaje para la educación superior. En J. Paredes y A. de la Herrán (coords.), Cómo enseñar en el aula universitaria (47-58). Madrid: Pirámide.

Vázquez, E. (2014). Tareas 2.0 para el aprendizaje y evaluación de segundas lenguas en entornos virtuales de aprendizaje. Píxel-Bit. Revista de Medios y Educación, 44, 185-199.

Velandia-Mesa, C., Serrano-Pastor, F. J., y Martínez Segura, M. J. (2017). La investigación formativa en ambientes ubicuos y virtuales en Educación Superior. Comunicar, 51 (XXV), 9-18. DOI: https://doi.org/10.3916/C51-2017-01.

Wang, Y., y Sun, C. (2001). Internet-based real time language education: Towards a fourth generation distance education. CALICO Journal, 18 (3), 539-561.

La didáctica de la lengua en entornos virtuales de aprendizaje: el caso concreto de la enseñanzaaprendizaje del español como lengua extranjera y la plataforma Eleclips. E. Álvarez Ramos. Página 19 de 20 
Ware, P. (2005). "Missed communication" in online communication: Tensions in fostering successful online interaction. Language Learning \& Technology, 9 (2), 64-89.

Warschauer, M. (1996). Computer-assisted language learning: An introduction. En S. Fotos (ed.), Multimedia Language Teaching (3-20). Tokyo: Logos International.

Warschauer, M. (2000). Language, identity, and the Internet. En B. Kolko, L. Nakamura, y G. Rodman (eds.) Race in Cyberspace (151-170). Nueva York: Routledge.

Warschauer, M. (2002). A developmental perspective on technology in language education. TESOL Quarterly 36 (3), 453-475.

Warschauer, M. (2003). Demystifying the digital divide". Scientific American 289 (2), 42-47.

Warschauer, M. (2006). Literacy and technology: Bridging the divide. En D. Gibas y K.-L. Krause (eds.), Cyberlines 2: Languages and cultures of the Internet. (163174) Albert Park: James Nicholas.

Warschauer, M., Knobel, M., y Stone, L. (2004). Technology and equity in schooling: Deconstructing the digital divide. Educational Policy 18 (4), 562-588.

White, C. (2007). Innovation and identity in distance learning and teaching. Innovation in Language Learning and Teaching, 1, 97-110.

Wickham, A. (2014). Blended Learning: where are we? Modern English teacher, 23-3, 44-46.

Zapata-Ros, M. (2010). Evaluación de competencias en entornos virtuales de aprendizaje y docencia universitaria. RED. Revista de Educación a Distancia. Sección de Docencia Universitaria en la Sociedad del Conocimiento, 1, 1-34.

Zapata-Ros, M. (2015). Pensamiento computacional: Una nueva alfabetización digital. RED. Revista de educación a distancia, 46, 1-47.

La didáctica de la lengua en entornos virtuales de aprendizaje: el caso concreto de la enseñanzaaprendizaje del español como lengua extranjera y la plataforma Eleclips. E. Álvarez Ramos. Página 20 de 20 